\title{
Severe mitral regurgitation due to mitral leaflet aneurysm diagnosed by three-dimensional transesophageal echocardiography: a case report
}

Takao Konishi ${ }^{1,3^{*}}$, Naohiro Funayama ${ }^{1}$, Tadashi Yamamoto ${ }^{1}$, Daisuke Hotta ${ }^{1}$, Kenjiro Kikuchi ${ }^{1}$, Katsumi Ohori ${ }^{2}$, Hiroshi Nishihara ${ }^{3}$ and Shinya Tanaka ${ }^{3}$

\begin{abstract}
Background: A small mitral valve aneurysm (MVA) presenting as severe mitral regurgitation (MR) is uncommon.

Case presentation: A 47-year-old man with a history of hypertension complained of exertional chest discomfort. A transthoracic echocardiogram (TTE) revealed the presence of MR and prolapse of the posterior leaflet. A 6$\mathrm{mm}$

in diameter MVA, not clearly visualized by TTE, was detected on the posterior leaflet on a three-dimensional (3D) transesophageal echocardiography (TEE). The patient underwent uncomplicated triangular resection of P2 and mitral valve annuloplasty, and was discharged from postoperative rehabilitation, 2 weeks after the operation. Histopathology of the excised leaflet showed myxomatous changes without infective vegetation or signs of rheumatic heart disease.
\end{abstract}

Conclusions: A small, isolated MVA is a cause of severe MR, which might be overlooked and, therefore, managed belatedly. 3D TEE was helpful in imaging its morphologic details.

Keywords: Mitral leaflet aneurysm, Mitral regurgitation, Three-dimensional transesophageal echocardiography, Case report

\section{Background}

Mitral valve aneurysm (MVA), a rare disorder first described by Saphir and Leroy in 1948 [1], is usually a complication of infective endocarditis [2-7]. Other causes of this disorder are rheumatic heart disease, Marfan's syndrome [8], aortic regurgitation and hypertrophic cardiomyopathy. Furthermore, the reports of surgical case have usually described MVA 15 to $30 \mathrm{~mm}$ in diameter [5, 7, 9-14]. The complications of MVA includes expansion, perforation and severe valvular

\footnotetext{
* Correspondence: takaokonishi0915@gmail.com

'Department of Cardiology, Hokkaido Cardiovascular Hospital, 1-30, West 13, South 27, Chuou-ku, Sapporo 064-8622, Japan

${ }^{3}$ Department of Cancer Pathology, Hokkaido University, Graduate School of Medicine, Sapporo, Japan

Full list of author information is available at the end of the article
}

regurgitation. An early diagnosis followed by surgical treatment is of critical importance to reduce the rate of these complications. We report a 6-mm in diameter, isolated aneurysm of a mitral leaflet in a man who presented with severe mitral regurgitation (MR).

\section{Case presentation}

A 47-year-old man with a 10-year history of hypertension complained of exertional chest discomfort. On physical examination, he was alert, his height and body weight were $168.5 \mathrm{~cm}$ and $78 \mathrm{~kg}$, respectively, body temperature $36.3{ }^{\circ} \mathrm{C}$, systemic blood pressure 152/ 
$82 \mathrm{mmHg}$ and heart rate $56 \mathrm{bpm}$. The percutaneous oxygen saturation on room air was $97 \%$. A III/VI systolic murmur was heard at the apex, radiating to the left axilla, without manifestation of cardiac decompensation. He had no sign of Marfan's syndrome. The laboratory tests revealed a $4.0 \times 10^{3} / \mathrm{mm}^{3}$ white blood cell count with $66 \%$ granulocytes and $27 \%$ lymphocytes, a $20.4 \times 10^{4} / \mathrm{mm}^{3}$ platelet count, $15.7 \mathrm{~g} / \mathrm{dl}$ haemoglobin concentration, and $0.03 \mathrm{mg} / \mathrm{dl}$ C-reactive protein serum concentration. A chest roentgenogram showed a $48 \%$ cardiothoracic ratio, and the 12-lead electrocardiogram showed a high QRS voltage in the lateral precordial leads. A transthoracic echocardiogram (TTE) revealed MR with prolapse of the posterior leaflet (Fig. 1a-c) without vegetation or aortic regurgitation. The echocardiographic left atrial and left ventricular (LV) dimensions and LV ejection fraction are shown in Table 1A. A two-dimensional transoesophageal echocardiogram (TEE) showed mitral valve prolapse (Fig. 2a and c) and MR with a significant proximal isovelocity surface area (PISA) (Fig. 2b and d). On three-dimensional (3D) TEE, a 6-mm in diameter aneurysm was present on the posterior mitral valve leaflet, which was not clearly visible on TTE (Fig. 2e and Additional file: Video S1). 3D fullvolume colour TEE was also useful for illustrating the spatial relationship between the MVA and the MR jet (Fig. 2f and Additional file: Video S2). A LV angiogram (Fig. 3 and Additional file: Video S3) showed grade 3-4 MR and coronary angiograms showed no significant stenosis. The pressures measured during right heart catheterization are shown in Table 1B. Cardiac index was within normal limits (Table 1B).

The patient underwent open-heart, triangular resection of the P2 segment of the mitral valve and annuloplasty with a $28-\mathrm{mm}$ Séguin ring. Intraoperative inspection confirmed the presence of an aneurysm on the posterior mitral leaflet (Fig. 4a-c). High- and low-power histopathologic microphotographs showed myxomatous degeneration, without
Table 1 Transthoracic echocardiographic and right heart catheterisation measurements

\begin{tabular}{ll}
\hline A. Transthoracic echocardiogram & 61 \\
Left ventricular ejection fraction, \% & \\
Diameters, mm & 47 \\
Left atrial & 55 \\
Left ventricular (end-diastolic) & \\
B. Right heart catheterization & \\
Pressures, mmHg & $10(1-5)$ \\
Right & \\
Atrial & $36(15-30)$ \\
Ventricular & $4(1-7)$ \\
End-systolic & \\
End-diastolic & \\
Pulmonary & $35(15-30)$ \\
Arterial & $18(4-12)$ \\
Systolic & $24(9-18)$ \\
Diastolic & $18(4-12)$ \\
Mean & $3.2(2.5-4.0)$ \\
Capillary wedge &
\end{tabular}

Normal values are shown in parentheses

active mitral valve endocarditis or inflammatory cellular infiltration of the posterior leaflet (Fig. 5a-c). The postoperative course was uneventful and the chest discomfort resolved during the postoperative rehabilitation program. The patient was discharged from postoperative rehabilitation, 2 weeks after the operation, and has remained free from significant MR over a 2-year follow-up.

\section{Discussion}

This case illustrates the rare characteristics of an isolated, non-infective MVA, which, albeit small, caused severe MR. This observation, to the best of our
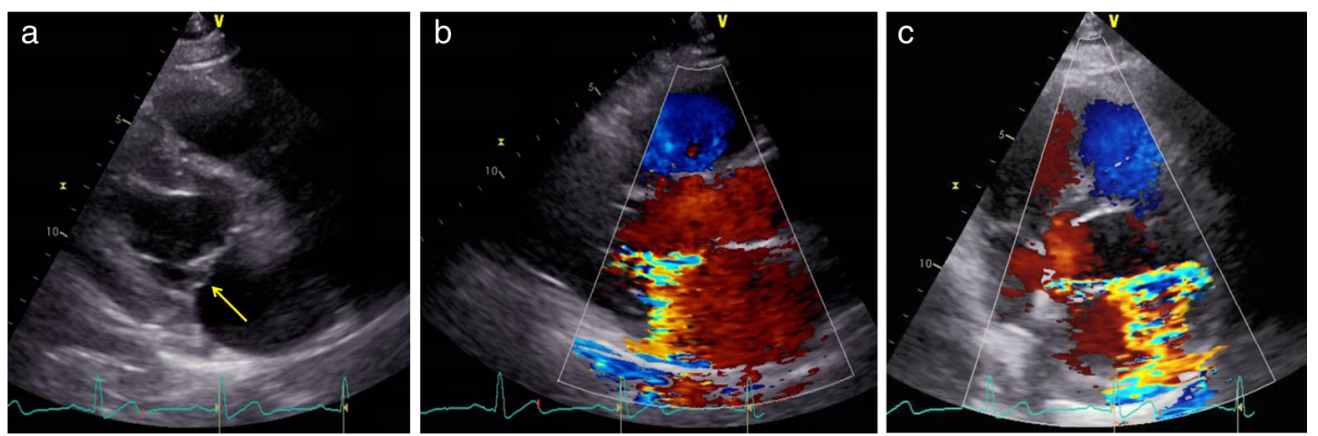

Fig. 1 Transthoracic echocardiogram. $\mathbf{a}, \mathbf{b}$ and $\mathbf{c}$. Parasternal long $\mathbf{a}$ and $\mathbf{b}$ and short $\mathbf{c}$ axis view of the posterior mitral valve leaflet slightly protruding into the left atrium (arrow) with severe eccentric mitral regurgitation 

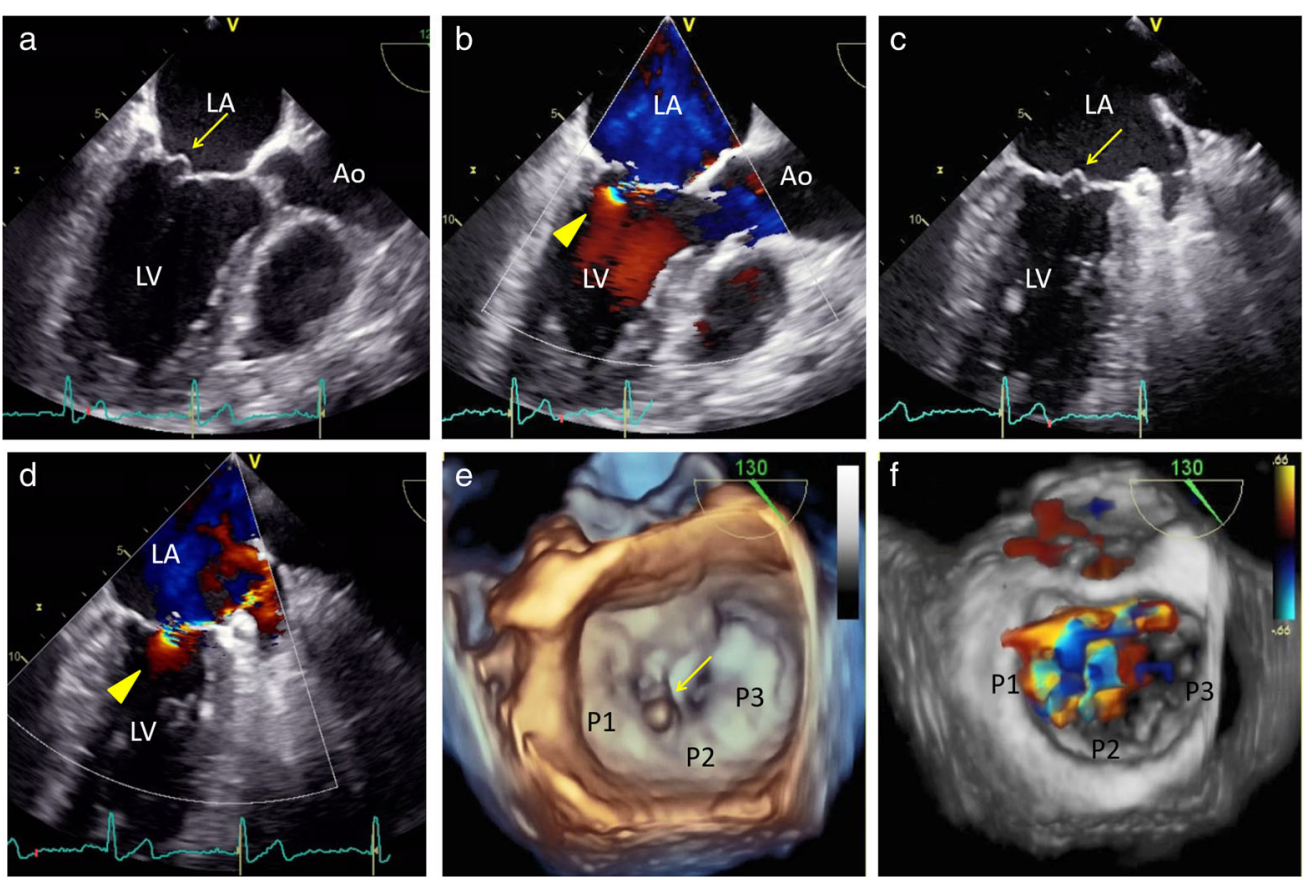

Fig. 2 Transoesophageal echocardiogram. a and c. Part of the posterior leaflet protrudes into the left atrium at a $129^{\circ}$ (a) and $63^{\circ}$ (c) angle in early systole (arrows). $L A=$ left atrium; $L V=$ left ventricle; $A O=$ Aorta. $\mathbf{b}$ and $\mathbf{d}$. The colour Doppler images shows a considerably large proximal isovelocity surface area (PISA) (arrowheads) and a prominently eccentric mitral regurgitation at a $129^{\circ}(\mathbf{b})$ and $63^{\circ}(\mathbf{d})$ angle in end-systole. e. Three-dimensional transoesophageal echocardiogram, observed from the left atrium towards the mitral valve annulus, showing the 6-mm in diameter aneurysm on the posterior leaflet (arrow). The aneurysm was located at mid-posterior leaflet (P2) near lateral-posterior leaflet (P1). f. Three-dimensional full-volume colour acquisition of the mitral valve illustrating the spatial relationship between the MVA and the MR jet

knowledge, has not been previously reported in the English language medical literature.

A MVA is a discrete bulge of the mitral leaflet toward the left atrium expanding during systole and collapsing during diastole [15]. Its formation is associated with congenital, structural diseases of the connective tissues,

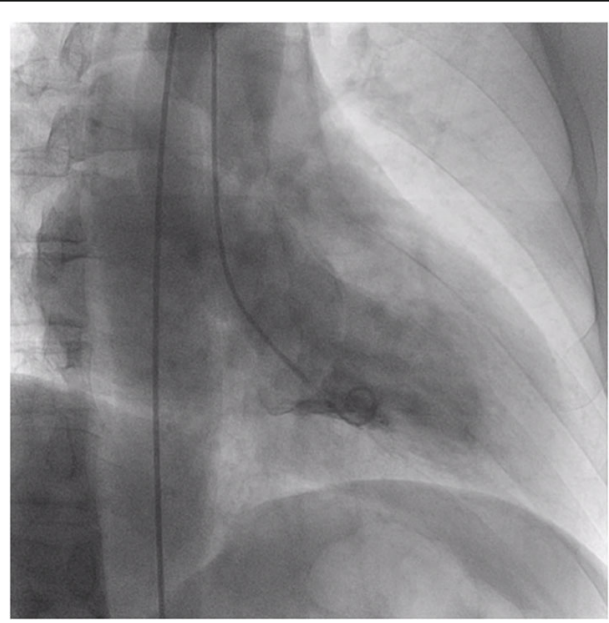

Fig. 3 Left ventriculography. Right anterior oblique, $30^{\circ}$ view of grade 3 to 4 mitral regurgitation (Seller's grading) including Marfan's syndrome [8], mitral valve prolapse [15], LV outflow tract obstruction, hypertrophic cardiomyopathy $[16,17]$ and bicuspid or quadricuspid aortic valves $[18,19]$. From these predisposing disorders, MVA is formed by acquired factors, such as rheumatic fever, Libman-Sachs endocarditis and, in most cases, infective endocarditis $[2,3,20-22]$. It is usually detected on the anterior leaflet, due to infective endocarditis caused by an aortic regurgitant jet striking the leaflet $[9,21,22]$. In this patient, however, it was on the posterior leaflet, in absence of regurgitant jet. In addition, the histopathology showed myxomatous degeneration without active endocarditis or inflammatory cells in the excised valve specimen (Fig. 5a-c). This supports our hypothesis that, in this patient, the prolonged hypertensive LV pressure overload, besides the congenital structural weakness secondary to myxomatous degeneration, caused the development of an isolated MVA of the posterior mitral leaflet in absence of infection.

The diagnosis of a small MVA, as was discovered in this patient, is often challenging, because its 3 dimensions are difficult to detect and identify on TTE. The superior performance of 3-dimensional TEE in clarifying the anatomical details of MVA has been reported [10, 23]. Since they may cause serious complications, such as systemic embolization, leaflet perforation, and recurrent infective 

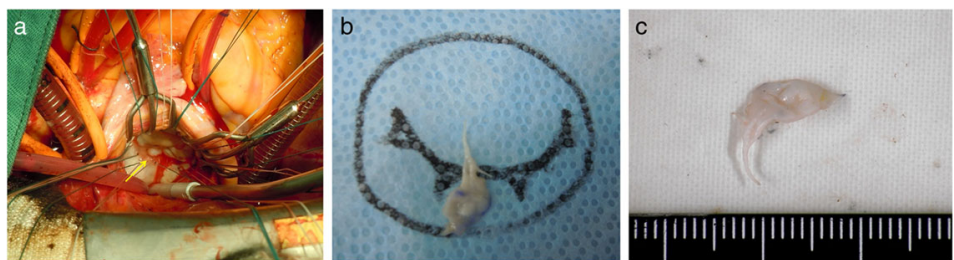

Fig. 4 Intraoperative aspect and histopathology of the posterior mitral valve leaflet. a. Intraoperative aspect of the MVA on the posterior mitral leaflet (arrow). b. The excised MVA was a part of the mid-posterior leaflet (P2). c. The excised MVA measured 6 mm in diameter

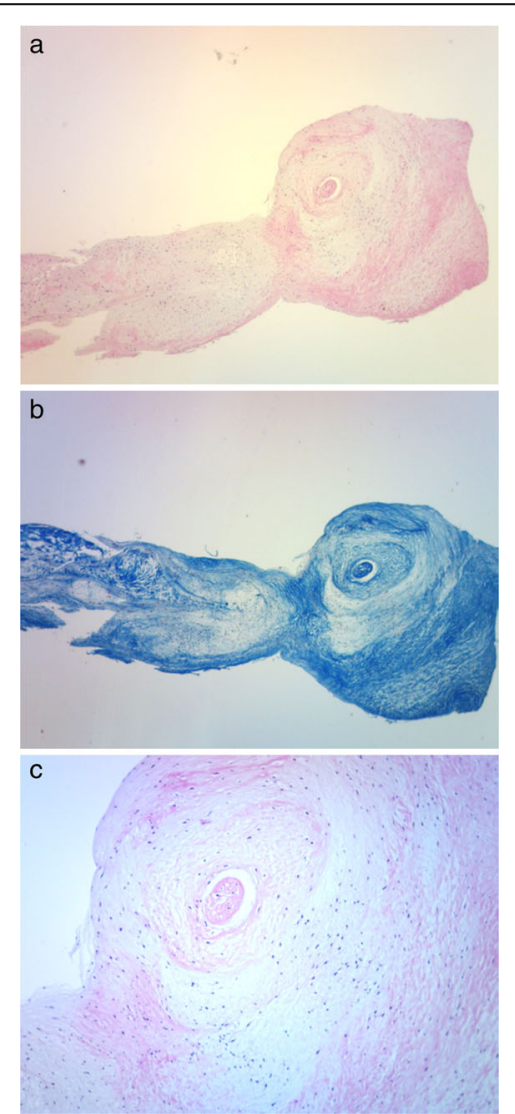

Fig. 5 Histopathology of the mitral valve aneurysm. a and b. Lowpower (X 5 original magnification) microphotograph of the mitral valve aneurysm showing prominent myxomatous changes observed after a haematoxylin and eosin, and $\mathbf{b}$ Masson Trichrome staining. c. Lowpower (X 10 original magnification) microphotograph showing no infiltration by inflammatory cells or active endocarditis in the posterior mitral leaflet

endocarditis [21, 24, 25], their prompt diagnosis and surgical management is of utmost importance. In this patient, 3dimensional TEE showed a small MVA, which could not be detected by TTE. This case also highlights the severity of MR caused by a MVA as small as $10 \mathrm{~mm}$ in diameter.

\section{Conclusions}

Small, isolated MVA causing significant MR are rare and may be neither visible on TTE nor managed in a timely fashion. Regardless of their size, MVA can cause severe MR. 3D TEE is an effective means of detecting their presence.

\section{Abbreviations}

3D: Three-dimensional; MR: Mitral regurgitation; MVA: Mitral valve aneurysm; TEE: Transesophageal echocardiography; TTE: Transthoracic echocardiography.

\section{Additional files}

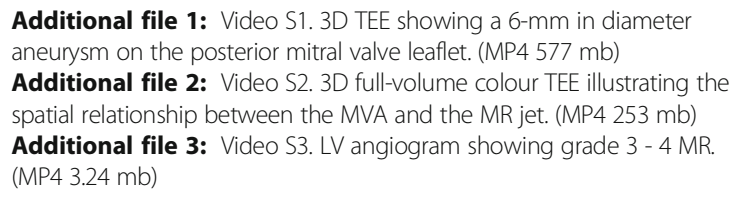

\section{Acknowledgments}

We thank Ms. Seiko Oono for her contribution in acquiring the echocardiographic images.

\section{Funding \\ None.}

\section{Availability of data and materials}

The datasets supporting the conclusions of this article are included within the article.

\section{Authors' contributions}

TK drafted the manuscript and prepared the figures. HN and ST analysed the histopathologic preparations. NF, TY, DH, KK and $\mathrm{KO}$ contributed to the composition of the manuscript. All authors have read and approved the submission of the final version of the manuscript.

\section{Competing interests}

The authors declare that they have no competing interests.

\section{Consent for publication}

Written informed consent was obtained from the patient for publication of this case report and any accompanying images and data.

\section{Ethics approval and consent to participate}

Research involving human data, in this report, was performed in accordance with the Declaration of Helsinki and was approved by Hokkaido Cardiovascular Hospital Ethics Committee.

\section{Author details}

${ }^{1}$ Department of Cardiology, Hokkaido Cardiovascular Hospital, 1-30, West 13, South 27, Chuou-ku, Sapporo 064-8622, Japan. ${ }^{2}$ Department of

Cardiovascular Surgery, Hokkaido Cardiovascular Hospital, Sapporo, Japan. 
${ }^{3}$ Department of Cancer Pathology, Hokkaido University, Graduate School of Medicine, Sapporo, Japan.

Received: 28 March 2016 Accepted: 15 November 2016 Published online: 22 November 2016

\section{References}

1. Saphir O, Leroy EP. True aneurysms of the mitral valve in subacute bacterial endocarditis. Am J Pathol. 1948;24(1):83-95.

2. Azevedo O, Ferreira F, Guardado J, Durães C, Quelhas I, Pereira A, et al. Mitral and aortic valve aneurysms secondary to infective endocarditis: impressive images of a rare echocardiographic finding. Eur J Echocardiogr. 2010;11(7):E28

3. Gao C, Xiao C, Li B. Mitral valve aneurysm with infective endocarditis. Ann Thorac Surg. 2004;78(6):2171-3.

4. Harada M, Hirai H, Inoue T, Sakai H, Lee T, Sugiyama Y, et al. Aortic and mitral valve aneurysms complicated with infective endocarditis: a case report. J Cardiol. 1998;31 Suppl 1:105-13.

5. Hotchi J, Hoshiga M, Okabe T, Nakakoji T, Ishihara T, Katsumata T, et al. Impressive echocardiographic images of a mitral valve aneurysm. Circulation. 2011;123(14):e400-2.

6. Michelena HI, Suri RM, Enriquez-Sarano M, Dearani J. Ruptured mycotic aneurysm of the mitral valve on real-time 3-dimensional transesophageal echocardiography. J Am Coll Cardiol. 2010;56(2):154.

7. Reid CL, Chandraratna AN, Harrison E, Kawanishi DT, Chandrasoma P, Nimalasuriya A, et al. Mitral valve aneurysm: clinical features, echocardiographic-pathologic correlations. J Am Coll Cardiol. 1983;2(3):460-4.

8. Edynak G, Rawson A. Ruptured aneurysm of the mitral valve in a Marfan-like syndrome. Am J Cardiol. 1963;11(5):674-677.

9. Northridge DB, Gnanapragasam JP, Houston AB. Diagnosis of mitral valve aneurysm by transoesophageal echocardiography. Br Heart J. 1991;65(4):227-8.

10. Al-Refai MA, Oueida FM, Lui RC, Al-Saif SM, Al-Omran HM. Impressive echocardiographic images of a rare pathology: Aneurysm of the mitral valve - Report of two cases and review of the literature. J Saudi Heart Assoc. 2012;25(1):47-51.

11. Anzouan-Kacou JB, Kramoh E, Coulibaly I. Perforated aneurysm of the anterior mitral valve. A rare cause of severe mitral regurgitation. Arch Cardiovasc Dis. 2010;103(4):275-6.

12. Subban V, Krishnamurthy V, Mohanraj A, Ajit MS. Posterior mitral leaflet aneurysm - a rare cause of mitral regurgitation. Indian Heart J. 2012;64(4):402-3.

13. Zhang $\mathrm{H}$, Chen $\mathrm{H}$, Sun $\mathrm{X}$, Yang $\mathrm{S}$, Wang $\mathrm{C}$. Repair for mitral valve aneurysm using autologous pericardium: a case of our experience. J Cardiothorac Surg. 2014;9:148.

14. Gajjar TP, Desai NB. True aneurysm of anterior mitral leaflet-a rare entity. J Thorac Cardiovasc Surg. 2012;144(3):e93-5.

15. Ruckel A, Erbel R, Henkel B, Kramer G, Meyer J. Mitral valve aneurysm revealed by cross-sectional echocardiography in a patient with mitral valve prolapse. Int J Cardiol. 1984;6(5):633-7.

16. de Castro S, Adorisio R, Pelliccia A, Papetti F, Fedele F, Pandian NG. Perforated aneurysms of left side valves during active infective endocarditis complicating hypertrophic obstructive cardiomyopathy. Eur J Echocardiogr. 2002;3(2):100-2.

17. Carpenter PM, Atai M, Hoit B. Aneurysm of the mitral valve in a patient with hypertrophic cardiomyopathy. Am J Cardiovasc Pathol. 1988;2(3):273-6.

18. Hori D, Tanaka M, Yamaguchi A, Adachi H, Ino T. Extracardiac aneurysm of the interleaflet triangle above the aortic-mitral curtain due to infective endocarditis of the bicuspid aortic valve. Gen Thorac Cardiovasc Surg. 2008; 56(8):424-6

19. Unger $P$, Preumont N, Stoupel E. Images in cardiology. Ruptured sinus of Valsalva aneurysm with right ventricular obstruction, quadricuspid aortic valve, and ventricular septal defect. Heart. 2000;84(4):424.

20. Takayama T, Teramura M, Sakai H, Tamaki S, Okabayashi T, Kawashima T, et al. Perforated mitral valve aneurysm associated with Libman-Sacks endocarditis. Intern Med. 2008:47(18):1605-8.

21. Kharwar RB, Mohanty A, Sharma A, Narain VS, Sethi R. Ruptured anterior mitral leaflet aneurysm in aortic valve infective endocarditis-evaluation by three-dimensional echocardiography. Echocardiography. 2014;31(3):E72-6.

22. Marcos-Alberca P, Rey M, Serrano JM, Fernandez-Rozas I, Navarro F, Contreras A, et al. Aneurysm of the anterior leaflet of the mitral valve secondary to aortic valve endocarditis. J Am Soc Echocardiogr. 2000; 13(11):1050-2
23. Edwards NC, Smith NL, Steeds RP. Beyond the artery and ventricle - an aneurysm of the mitral valve leaflet. Echocardiography. 2013;30(8):E258-9.

24. Vijay SK, Tiwari BC, Misra M, Dwivedi SK. Incremental value of threedimensional transthoracic echocardiography in the assessment of ruptured aneurysm of anterior mitral leaflet. Echocardiography. 2014;31(1):E24-6.

25. Lee CH, Tsai LM. Transesophageal echocardiographic recognition of mitral valve aneurysm. J Ultrasound Med. 2005;24(8):1141-4.

\section{Submit your next manuscript to BioMed Central and we will help you at every step:}

- We accept pre-submission inquiries

- Our selector tool helps you to find the most relevant journal

- We provide round the clock customer support

- Convenient online submission

- Thorough peer review

- Inclusion in PubMed and all major indexing services

- Maximum visibility for your research

Submit your manuscript at www.biomedcentral.com/submit
Biomed Central 